\title{
Integrin signaling downregulates filopodia during muscle-tendon attachment
}

\author{
Benjamin Richier ${ }^{1,2, *}$, Yoshiko Inoue ${ }^{1,2,3, *}$, Ulrich DobramysI ${ }^{1,2}$, Jonathan Friedlander ${ }^{1,3}$, Nicholas H. Brown ${ }^{1,3}$ \\ and Jennifer L. Gallop ${ }^{1,2, \pm}$
}

\begin{abstract}
Cells need to sense their environment to ensure accurate targeting to specific destinations. This occurs in developing muscles, which need to attach to tendon cells before muscle contractions can begin. Elongating myotube tips form filopodia, which are presumed to have sensory roles, and are later suppressed upon building the attachment site. Here, we use live imaging and quantitative image analysis of lateral transverse (LT) myotubes in Drosophila to show that filopodia suppression occurs as a result of integrin signaling. Loss of the integrin subunits $\alpha \mathrm{PS} 2$ and $\beta \mathrm{PS}$ (also known as If and Mys, respectively, in flies) increased filopodia number and length at stages when they are normally suppressed. Conversely, inducing integrin signaling, achieved by the expression of constitutively dimerised $\beta$ PS cytoplasmic domain (di $\beta$ ), prematurely suppressed filopodia. We discovered that the integrin signal is transmitted through the protein $\mathrm{G}$ protein-coupled receptor kinase interacting $\operatorname{ArfGAP}(\mathrm{Git})$ and its downstream kinase p21-activated kinase (Pak). Absence of these proteins causes profuse filopodia and prevents the filopodial inhibition mediated by di $\beta$. Thus, integrin signaling terminates the exploratory behavior of myotubes seeking tendons, enabling the actin machinery to focus on forming a strong attachment and assembling the contractile apparatus.
\end{abstract}

KEY WORDS: Filopodia, Integrin signaling, Actin dynamics, Muscle, Drosophila

\section{INTRODUCTION}

Muscle development involves similar phases of differentiation and movement in Drosophila and vertebrate embryos (Schweitzer et al., 2010). Drosophila somatic muscles display a stereotyped pattern of 30 muscles per abdominal hemisegment (Fig. 1A, $\mathrm{A}^{\prime}$ ). The lateral transverse (LT) muscles migrate and elongate towards the epidermis, and their attachment sites, near the surface, are particularly accessible for live imaging (Fig. 1A, $\mathrm{A}^{\prime}$ ). Each LT muscle is a single syncytial cell, the myotube, which is formed by fusion of fusion-competent myoblasts to a founder cell (Rochlin et al., 2010). Each founder cell

\footnotetext{
${ }^{1}$ The Gurdon Institute, Tennis Court Rd, Cambridge CB2 1QN, UK. ${ }^{2}$ Dept. of Biochemistry, University of Cambridge, Cambridge CB2 1GA, UK. ${ }^{3}$ Dept. of Physiology, Development and Neuroscience, University of Cambridge, Cambridge CB2 3DY, UK.

${ }^{*}$ These authors contributed equally to this work

${ }^{\ddagger}$ Author for correspondence (j.gallop@gurdon.cam.ac.uk)
}

(D) B.R., 0000-0001-7641-8508; Y.I., 0000-0002-9709-8600; U.D., 0000-00019363-654X; J.F., 0000-0003-3796-9571; N.H.B., 0000-0002-8958-7017; J.L.G., 0000-0002-9978-1382

This is an Open Access article distributed under the terms of the Creative Commons Attribution License (http://creativecommons.org/licenses/by/3.0), which permits unrestricted use, distribution and reproduction in any medium provided that the original work is properly attributed.

Received 26 February 2018; Accepted 12 July 2018 contains the transcriptional instructions for the development of that particular muscle. This includes the number of fusion events and the sites of attachment to the epidermis (Fig. 1B-B") (de Joussineau et al., 2012). While some of the guidance cues known to target myotubes to their tendon cells overlap with those guiding neuronal cell migration (Slit, Robo and Derailed), some cues are unique to specific myotubes, such as the transmembrane protein Kon-tiki in ventral-longitudinal muscles (Callahan et al., 1996; Estrada et al., 2007; Kramer et al., 2001; Schnorrer et al., 2007). Myotube migration and tendon cell specification proceed in concert to organize muscle architecture (Schweitzer et al., 2010) with, for adult indirect flight muscles, extensive protrusions occurring in both cell types at the time of attachment (Weitkunat et al., 2014).

In the embryo, the myotube ends that move toward the tendon cells produce actin-rich finger-like protrusions known as filopodia (Schnorrer and Dickson, 2004), which have been implicated in the ability of cells to find their correct location (Davenport et al., 1993; Jacquemet et al., 2015). Once at the right locations, the myotubes need to stop migrating, otherwise secure attachments cannot form and myotubes 'overshoot' the tendon cells. Such a phenotype has been observed in Drosophila deficient in G protein-coupled receptor kinase interacting ArfGAP (Git) (Bahri et al., 2009). At tendon cells, myotubes make a strong integrin-based attachment, protrusions reduce and the myotube tips become rounded. There is concerted assembly of the contractile actin machinery to form the sarcomeres, and tension itself is implicated in this process (Weitkunat et al., 2014). This developmental process illustrates the exquisite intracellular specificity of the actin cytoskeleton, with appropriate actin filament nucleators, elongators and bundlers recruited to different cell areas in a spatially and temporally coordinated manner. Remodeling of the actin cytoskeleton is proposed to occur actively, for example from the activation of Rho-type GTPases or phosphorylation cascades. Within the context of myotube migration, one could also envisage that the building of the extensive contractile machinery of the myotube could lead to a natural reduction in the migratory, protrusive actin activity instead of being actively suppressed at the appropriate time.

In this study, we investigate the role of integrins in the regulation of filopodia during the formation of the muscle-tendon attachment site. We find that, as the muscle reaches the tendon cell, integrins have two important activities: their well characterized role in attaching to the tendon cell via an intervening extracellular matrix, and a new function mediating a signaling pathway that actively suppresses filopodia. We characterize Git and its downstream target p21-activated kinase (Pak) as components of this pathway. The transition from migration to muscle-tendon attachment provides a key example within a whole organism, where the contribution of integrins demonstrably involves signaling in addition to forming adhesions. Thus, integrin signaling helps to provide a migratory stop signal that acts in conjunction with the formation of an integrin adhesion junction at the muscle ends. 

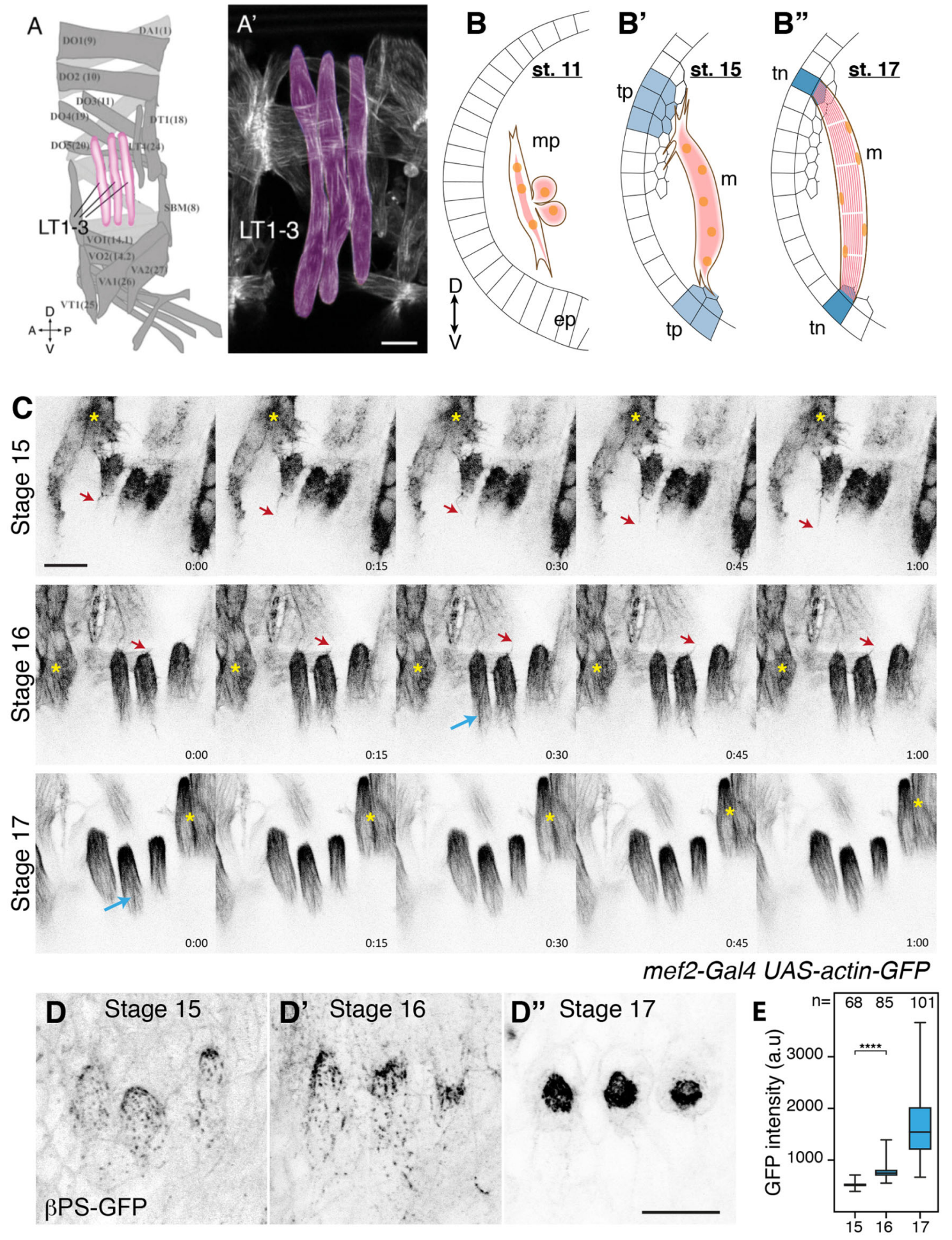

Fig. 1. Filopodia dynamics in embryonic LT muscles. (A,A') Schematic of the stereotypical muscle pattern in segments $A 2$ to $A 7$ (Ruiz-Gómez et al., 1997). Magenta, lateral transverse muscles (LT1-3); light gray, interior muscles; gray, other muscles. (A') Mature LT muscles (magenta) in a stage 17 embryo visualized by mef2-Gal4 UAS-GFP-actin. (B-B") Schematic of developmental steps of Drosophila myogenesis (embryo cross-section). (B) Stage 11: fusion-competent myoblasts fuse to one founder cell to form a multinucleate myotube progenitor $(\mathrm{mp})$. ( $\left.\mathrm{B}^{\prime}\right)$ Stage 15: the leading edge of extending myotubes form filopodia, searching for their targets, the tendon precursors (tp) located in the epithelial layer (ep). (B") Stage 17: filopodia formation ceases, and both ends of the myotube attach to terminally differentiated tendon cells (tn). (C) Time-lapse images of LT myotube tips at stages 15,16 and 17 . Filopodia form during stage 15 and 16 but appear smaller at later stages (red arrows). Within the myotube, actomyosin fibers develop during stage 16 (blue arrows). Yellow asterisks, adjacent myotubes. Time is given in min:s. (D-D") $\beta P S-G F P$ expressed at endogenous levels, revealing integrin accumulation at the muscle attachment site at stages 15,16 and 17 . (E) Quantification of the $\beta P S-G F P$ intensity at myotube tips (8-10 embryos). The box represents the 25-75th percentiles, and the median is indicated. The whiskers show the range. ${ }^{* * *} P=2.2 \times 10^{-16}$ (Mann-Whitney test). Orientation is as indicated in $A$ and $B$ [dorsal $(D)$, ventral $(V)$, anterior $(A)$ and posterior $\left.(P)\right]$. Scale bars: $10 \mu \mathrm{m}$. 


\section{RESULTS AND DISCUSSION \\ Filopodia at the myotube leading edges are downregulated when the attachment with the tendon cell is formed}

Myotubes migrate towards prospective tendon cells in the epidermis and form stable attachment sites. Contractile actomyosin bundles then generate muscle contraction and embryo movement (Schweitzer et al., 2010) (Fig. 1A-B"). By observing expression of GFP-actin in the dorsal tip of lateral transverse (LT) muscles (using the muscle-specific Gal4 driver mef2-Gal4) in live embryos, we visualized filopodial dynamics (Fig. 1C, red arrows; Movies 1-3). Dorsal LT tips were very close to each other with active filopodia during stage 15 and early stage 16 ( $\sim 3 \mathrm{~h}$, Fig. 1C, stage 15$)$. As development progressed, LT tips became more spaced out, and the number and length of filopodia reduced while actin bundles formed instead within the myotube (Fig. 1C, blue arrows), with a rounded end of the myotube making the attachment (Fig. 1C, stage 16). At stage 17, filopodia had become very rare and muscle contractions started (Fig. 1C, stage 17). This apparent reduction of filopodia number at the same time that the attachment forms is consistent with previous observations in embryonic ventral oblique muscles (Bate, 1990; Schnorrer and Dickson, 2004). The integrin expressed by the muscles is the heterodimer $\alpha \mathrm{PS} \beta \mathrm{PS}$, which attaches the muscle to the tendon cell so that the force of muscle contractions is transmitted to the exoskeleton for movement (Maartens and Brown, 2015; Devenport et al., 2007). We observed, similar to what was seen in previous studies in indirect flight muscle development (Weitkunat et al., 2014), that filopodial downregulation and $\beta$ PS integrin accumulation at the attachment sites occurred simultaneously (Fig. 1D,E).

\section{Integrin signaling downregulates filopodia}

To test whether integrins are inhibiting filopodia formation, it was necessary to quantify and characterize the filopodial behavior produced by muscles LT1-3 (as shown in Fig. 1A). To do this, we developed an image segmentation and analysis pipeline to reconstruct filopodia in time-lapse movies from confocal microscopy $z$-stacks. The location of LT muscle tips within a complex tissue environment containing other muscles, and the extension of filopodia in many directions, made the analysis too complex for automated quantification software. We therefore employed a semi-automated pipeline consisting of three main steps: (1) automatic segmentation and extraction of filopodia segments, (2) manual editing to remove false detections and merging of segments from the same filopodium, and (3) automated linking of edited filopodia across time points (Fig. $\mathrm{S} 1$ shows example fits). The pipeline outputs a list of filopodia and their coordinates for each time point of their existence. The reconstruction of very small filopodia was very noisy; therefore, we excluded them to focus on filopodia that reached a maximum length of at least $3 \mu \mathrm{m}$ and existed for at least two time frames separated by 15 seconds. We then extracted the maximum length and the average number of filopodia (a $z$ projection is shown in Fig. 2A, Movie 1). We quantified the filopodia for the three LTs together, rather than individually, as the three myotube tips are closely apposed and difficult to separate. Because we label all muscles, adjacent muscles (Fig. 2, yellow asterisks) can mask LT muscle tips or their filopodia (Fig. 2, red arrows) in $z$ projection images, but the use of original $z$-stacks in the pipeline avoids this issue. Using this method, we validated that a strong reduction in filopodia number occurs from stage 15 to stage 16 in control embryos (Fig. 2D).

With this tool at hand, we visualized GFP-actin in embryos lacking the integrin $\beta$ PS subunit (myospheroid ${ }^{X G 43}$, mys $^{X G 43}$ ). In mys $^{X G 43}$ mutant myotubes, filopodia were resistant to the downregulation usually observed by stage 16 (Fig. 2A', $\mathrm{B}^{\prime}, \mathrm{D}$; Movie 4). A similar effect was observed in embryos lacking the integrin $\alpha$ PS2 subunit (inflated $d^{B 4}, i f^{B 4}$ ) although further defects in muscle development prevented accurate reconstruction (Fig. S2; Movie 5). inflated is the only $\alpha \mathrm{PS}$ subunit expressed in muscles, whereas both $\alpha \mathrm{PS} 1$ (encoded by mew) and $\alpha \mathrm{PS} 2$ are expressed in tendon cells (Devenport et al., 2007). These results suggest that integrins are required within the muscles to regulate filopodia at myotube tips. To distinguish between the signaling and adhesion functions of integrins, we used di $\beta$, a chimeric protein in which the intracellular domain of $\beta \mathrm{PS}$ is fused to the extracellular and transmembrane domains of a constitutively active form of the receptor Torso (Martin-Bermudo and Brown, 1999). This fusion protein constitutively dimerizes the cytoplasmic domain of the $\beta P S$ subunit, and mimics integrin signaling, even in the absence of integrin-mediated adhesion to the extracellular matrix (MartinBermudo and Brown, 1999). The expression of di $\beta$ in muscles prematurely reduced filopodia number in stage 15 embryos to a level similar to that seen at stage 16 in control embryos (Fig. 2C,D; Movie 6). Expression of this construct only in muscle cells also shows that the effect of integrin on filopodia suppression is cell autonomous rather than due to contributions from tendon cells.

The finding that filopodia number was affected by both integrin loss and gain of function suggests an active role for integrin signaling in filopodia formation. To get further insights into this mechanism, we also analyzed the maximum length reached by each filopodia in our dataset. Maximum lengths of LT muscle filopodia follow an approximately exponential distribution (a high number of small filopodia and gradually fewer longer ones) (Fig. S3). This distribution is indicative of stochastic incorporation of actin to generate filopodial length rather than generation of a preferred length. We display this as a normalized cumulative frequency chart (Fig. 2E) to make the differences between conditions easily visible. Similar to the reduction in numbers of filopodia, the lengths of filopodia were also reduced as the embryo develops from stage 15 to stage 16 (Fig. 2E; the line shifts to the left, showing proportionally fewer long filopodia). The filopodia in $m y s^{X G 43}$ mutants at stage 15 were similar to controls, but at stage 16 the reduction in length of filopodia failed to occur (the stage $16 m y s^{X G 43}$ line overlays that for the stage 15 controls). Conversely, when di $\beta$ was expressed in muscles, the maximum lengths distribution of stage 15 overlapped with the stage 16 control (Fig. 2E). This shows that dominant-active integrin signaling caused a premature reduction of the filopodia maximum length. This measure of filopodia length provides an independent quantification from filopodia number, hinting that integrin signaling may be affecting multiple points of actin regulation, affecting both the number and morphology of the resulting filopodia. We used live imaging of integrin and the membrane in an effort to discover the location of the integrin signaling. We detected faint levels of integrin within filopodia, intermediate levels at the base of some, but not all, filopodia, and high levels in dots separated from the filopodia (Fig. 2F-J). In 3D, the muscle tip is reminiscent of a fingertip pressed on a surface (the basal epidermis), with integrin adhesions at the fingerprint level (Fig. 2G-I) and filopodia at fingernail level (Fig. 2I-J). We did not detect any correlation between the integrin distribution and the presence or absence of filopodia, so it is not clear where signaling occurs.

\section{Git and Pak control filopodia formation downstream of integrins}

We next investigated the signals and pathways downstream of integrins in LT muscles during attachment to the tendon. The 

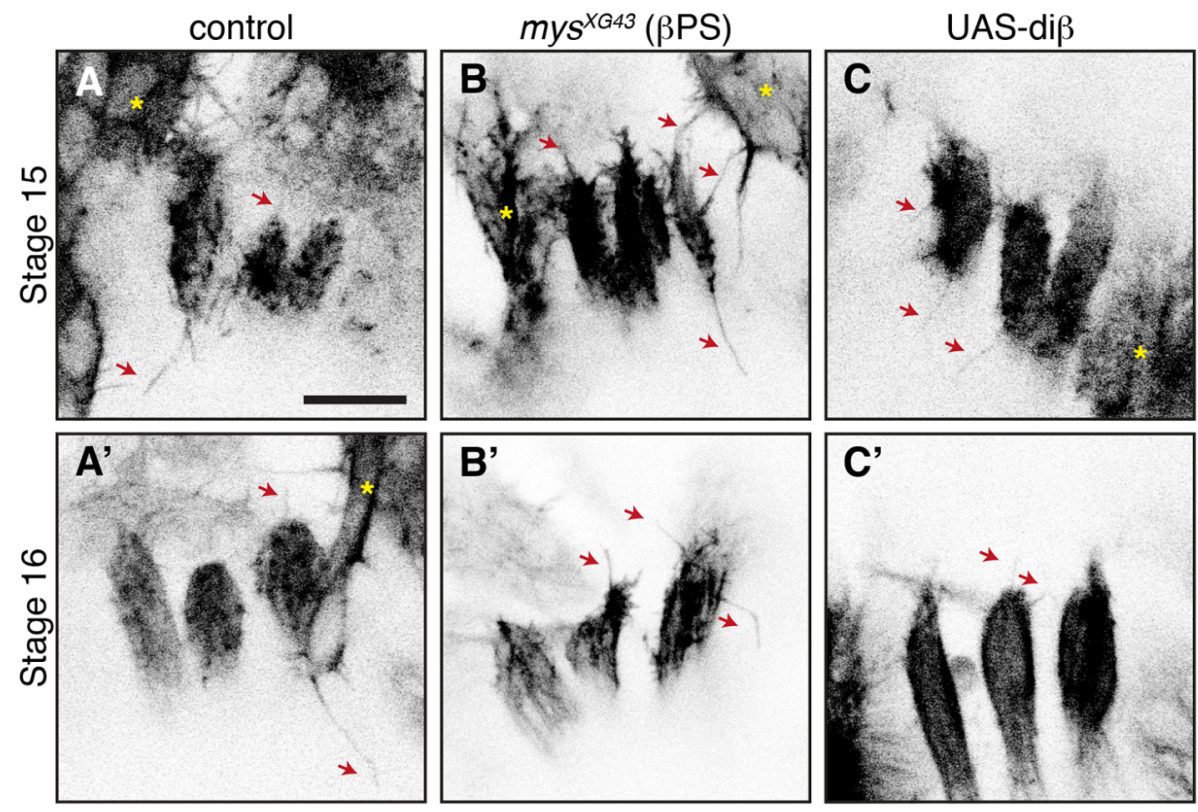

mef2-Gal4 UAS-GFPactin
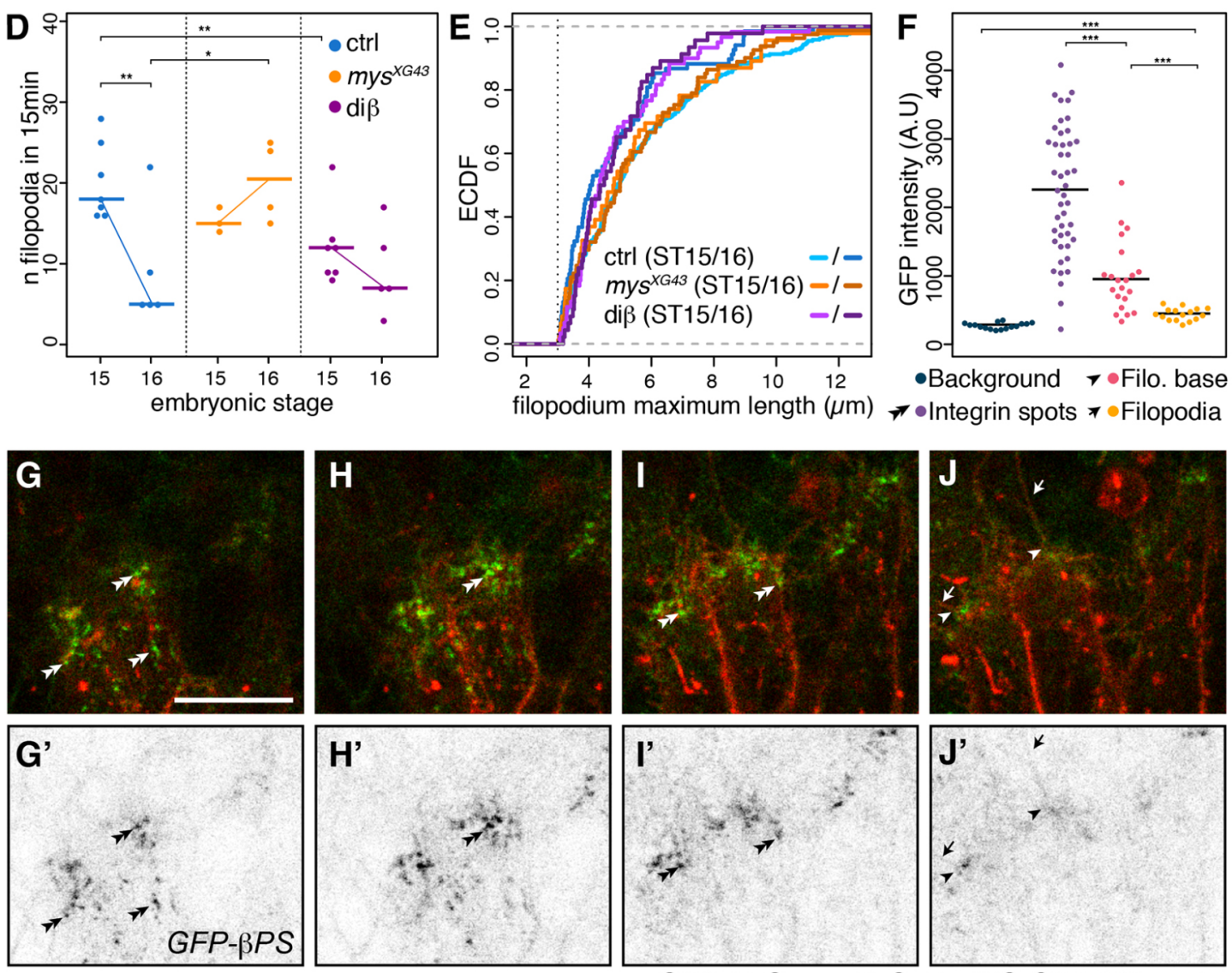

GFP-BPS;; mef2-Gal4 UAS-CD4-tdTomato

Fig. 2. Integrins regulate filopodia formation at LT muscle tips. (A-C) Still z projections from time-lapse movies showing control (A,A'), zygotic $\beta P S$ mutant mys $^{X G 43}\left(B, B^{\prime}\right)$ and UAS-di $\left(C-C^{\prime}\right)$ LTs at stage 15 and 16. Filopodia are marked with red arrows. Yellow asterisks, adjacent muscles. (D) Number of filopodia at LT tips during initiation and maturation of attachment site in a 15-min time window. Graph showing individual values (dots) and median (horizontal bars). Thin lines between stages illustrate the change in filopodia number. ${ }^{*} P<0.025$, ${ }^{* *} P<0.01$. (E) We apply a cut-off at $3 \mu \mathrm{m}$ to discard the shortest structures and show an empirical cumulative distribution function (ECDF) of the maximum length values. Each point on the curve indicates the proportion of filopodia with a given maximum length or less. The curve for ctrl at stage (ST)16 shifts towards the left compared to ctrl at ST15, indicating that the proportion of long filopodia is smaller at stage 16. (F-J') $\beta P S-G F P$ in LT tips (stage 15 embryos) in four focal planes (0.5 $\mu \mathrm{m}$ distance) (G-J). $\beta P S-G F P$ integrin (green) localizes at the membrane (red, mef-Gal4 UAS-CD4tdTomato), including within filopodia (arrows), filopodia base (arrowheads) and strongly at focal adhesions (double arrowheads). (F) GFP intensity quantification ( $n=3$ embryos). ${ }^{* * *} P<0.001,{ }^{* * * *} P<0.0001$ (see Table S1). Scale bars: $10 \mu \mathrm{m}$. 
protein Git has been implicated in the integration and transduction of various signals at the membrane, including integrins and receptor tyrosine kinases (Hoefen and Berk, 2006). Git recruits Pak to sites of focal adhesions, as part of its complex with Pak-interacting exchange factor (PIX), and at muscle ends (Bahri et al., 2009; Hoefen and Berk, 2006). Moreover, Git mutants have previously been reported to have defects in muscle patterning and targeting in ventral muscles (Bahri et al., 2009). In these mutants, the muscles target and attach inappropriately, suggesting a defect in myotube tips sensing tendon cells.

In embryos lacking both maternal and zygotic contributions of Git (Git mz), LT myotubes displayed a more elongated and pointed tip instead of the rounded shape observed in wild-type embryos both at stage 15 and 16 (Fig. 3A,E'), and the reduction in filopodia number at stage 16 failed to occur (Fig. 3F). As with loss of integrin, the filopodia appeared longer than in controls, but also more branched (Fig. 3A, $\mathrm{A}^{\prime}$, blue arrows). In addition, large protrusions, similar to lamellipodia, formed at muscle ends (Fig. 3A, $\mathrm{A}^{\prime}$, red arrowheads; Movie 7), which unfortunately prohibited an accurate quantification of filopodia length. A very similar phenotype was observed in embryos lacking both maternal and zygotic contributions of Pak (Pak mz) (Fig. 3B,B'; Movie 8). Thus, Git and its downstream effector Pak are also required to suppress filopodia at stage 16 and have an additional role in regulating the morphology of actin protrusions. The appearance of these larger protrusions when muscles lack Git or Pak suggests that there is normally a basal level of signaling through Git to suppress these structures. This is consistent with defects caused by the absence of Git in ventral muscles prior attachment, at stage 14-15 (Bahri et al., 2009).

To test whether Git and Pak act downstream of integrins, or via parallel pathways, we expressed di $\beta$ in embryos lacking Git or Pak. The ability of di $\beta$ to prematurely suppress filopodia at stage 15 was lost in the absence of Git or Pak (Fig. 3C,D,F; Movies 9,10). The number of filopodia in embryos deficient in Git or Pak and expressing di $\beta$ were somewhat elevated further than either Git or Pak alone (however, only one of the comparisons reached statistical significance). Di $\beta$ also exerts dominant negative effects on adhesion (Tanentzapf et al., 2006), which may allow increased filopodia in the absence of an inhibitory signal. Overall, we conclude that Git and Pak act downstream of integrin signaling to suppress filopodia.

\section{Conclusions}

During morphogenesis, cells must simultaneously integrate several signals and adapt their morphology in coordination with their environment. During their attachment to tendon cells, myotubes need to recognize their target partner, stop elongating, start forming an adhesion and prepare the actomyosin structures that will produce muscle contractions in a short time. We showed that filopodia, which can be used to explore and identify tendon cells and are involved in myotube elongation, are actively suppressed during muscle attachment. Integrins, which accumulate at the myotube tip to form a strong adhesion with tendon cells, also provide a signal in parallel, acting through Git and Pak proteins. This is needed for the regulation of filopodia formation in order to ensure correct myotube
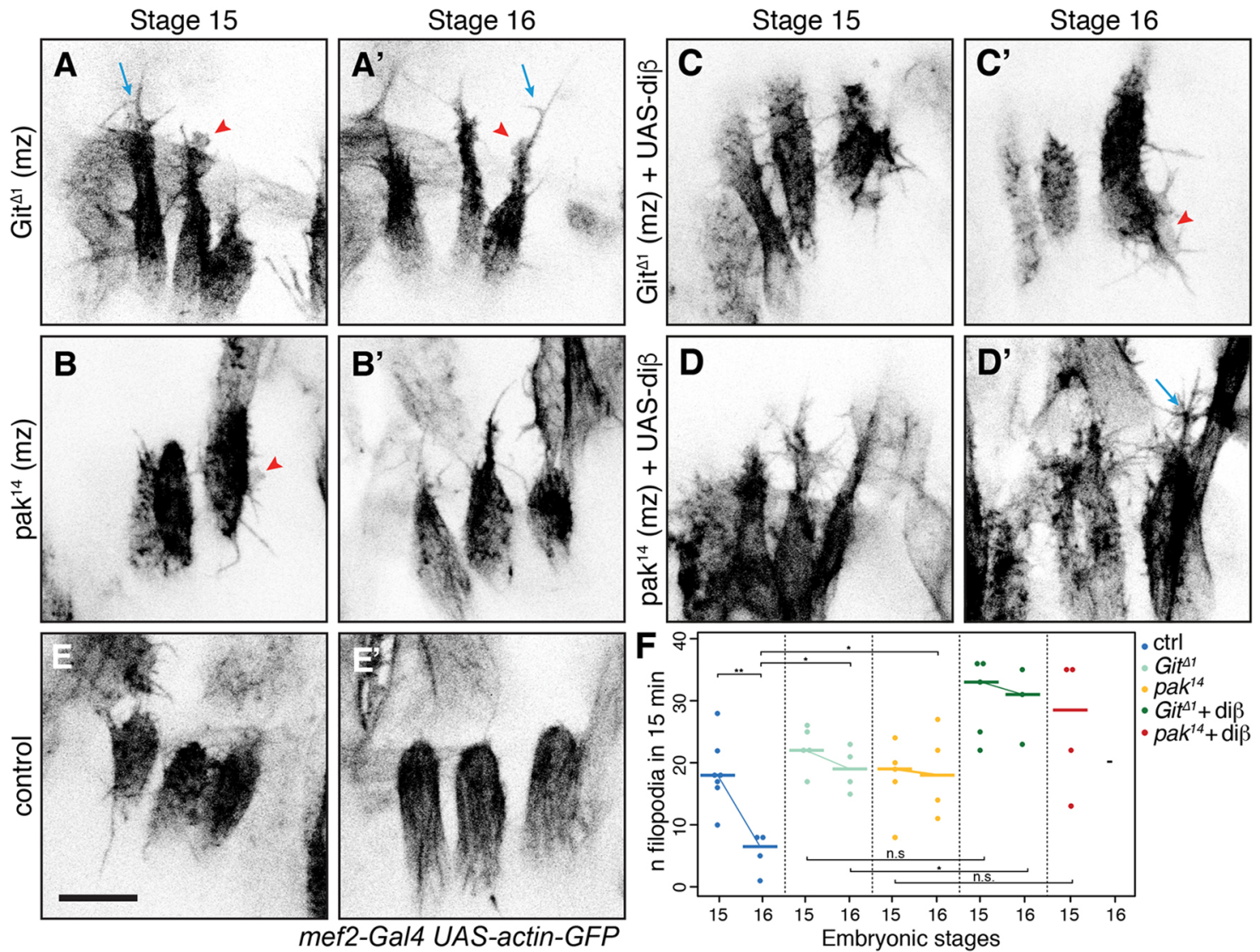

Fig. 3. Integrins act upstream of Git and Pak to suppress filopodia activity. Z projections for $\mathrm{Git}^{\Delta 1}\left(A, A^{\prime}\right), p^{2} k^{14}\left(B, B^{\prime}\right)$, di $\beta$ expression in $G i t^{\Delta 1}$ mz $\left(C, C^{\prime}\right)$ and pak $^{14} \mathrm{mz}\left(\mathrm{D}, \mathrm{D}^{\prime}\right)$ and control $\left(\mathrm{E}, \mathrm{E}^{\prime}\right)$. Blue arrows, branched filopodia; arrowheads, lamellipodia-like structures. Scale bar: $10 \mu \mathrm{m}$. (F) Quantification of filopodia number at dorsal LT muscle tips (Pak ${ }^{14}+$ di $\beta$ stage 16 , not enough values). Horizontal bars are the median. ${ }^{*} P<0.025,{ }^{* *} P<0.01$ (see Table S1). 
arrest and adhesion to tendon cells. It is likely that multiple proteins within the cytoskeletal machinery are targeted by Git and Pak, possibly through direct phosphorylation or indirect regulation. Ena, the Scar/WAVE complex and fascin are all known actin regulators that are sensitive to phosphorylation state and could be such targets (Comer et al., 1998; Loureiro et al., 2002; Mendoza, 2013; Zanet et al., 2012; Zeng et al., 2017). To conclude, our results demonstrate that filopodia suppression occurs via integrin signaling within the myotube, providing a valuable new paradigm for integrin signaling in Drosophila and the roles of integrins in filopodia regulation.

\section{MATERIALS AND METHODS \\ Drosophila stocks}

Flies were raised and crossed at room temperature. The wild-type strain used was white [1118]. Mef2-Gal4 was used to drive expression of these UAS constructs: $\quad w^{*} ; \quad P\{U A S-p G F P . A c t 88 F\} 1-2 \quad$ (BL9254), $w^{*} ; \quad P\{U A S$ pGFP.Act5C\}2-1 (BL 9258), w*; P\{UAS-CD4-tdTomato $\}$ (BL35837) all from the Bloomington stock center, and UAS-di $\beta$ (Martin-Bermudo and Brown, 1999). The genomic rescue construct used in this study was: $\beta P S$ GFP (Klapholz et al., 2015). The mutant lines include: mys [XG43] (Bunch et al., 1992), if [B4] (Brown, 1994), Git[41] (in this study) and pak[14] (Newsome et al., 2000; Bloomington stock center stock BL9123). Git [A1] and pak [14] germline clones were generated using FRTG13 Git [AI] and FRT82B pak[14] flies individually, essentially as described previously (Chou and Perrimon, 1996).

\section{Mutagenesis}

The fly line carrying the P-element insertion $P\{E P g y 2\} E Y 09254$ was used to isolate mutations in Git. Mobilization of the P-element was achieved by using the immobile element $P[r y+\Delta 2-3](99 B)$ as a transposase source (Robertson et al., 1988). The genotype of the transposase stock is $\mathrm{Sp} / \mathrm{CyO}$; $P[r y+\Delta 2-3](99 B) / T M 6$. Single jump starter males of the genotype $P\{E P g y 2\} E Y 09254 / C y O ; P[r y+\Delta 2-3](99 B) /+$ were crossed to second chromosome balancer females. From each cross, three white-eyed male progeny were crossed individually to a deficiency $D f(2 R) \operatorname{Stan} 2, F R T 42 D$ / $\mathrm{CyO}$ to screen for lethality. The Git alleles were analyzed by PCR for mapping of lesions in the Git locus. The excision allele Git [ $\Delta 1]$ deletes 1472 bp including the ATG and the first exon ( -520 to 952 relative to the first ATG of the Git gene).

\section{Live imaging of Drosophila embryos}

Dechorionated embryos (washed in 50\% bleach) were mounted on a glassbottomed dish with heptane glue and covered with water. In some cases, the genotype of embryos was determined by the absence of YFP-marked chromosome balancer prior to and after the recording. Live imaging was performed on an inverted Leica TCS-SP5 equipped with a $63 \times 1.4$ NA Plan Apo oil immersion objective or an Olympus FV-1000 confocal laser scanning microscope equipped with a $60 \times 1.3$ NA Plan Apo oil immersion lens at room temperature. To follow filopodia movement, we visualized stage 15-17 embryos in the indicated genetic backgrounds. Time-lapse movies of LT tips in embryos were recorded by taking $z$-stacks of 5-7 z sections $(0.7 \mu \mathrm{m}$ spacing $)$ at 15 -s intervals for $15 \mathrm{~min}$. For experiments, movies were recorded from three to five embryos per genotype, from different segments when recorded on the same embryos.

\section{Data analysis}

The segmentation pipeline takes every $z$-stack acquired throughout an imaging session separately. It outputs filopodia segments that are then edited by a user, followed by an automatic linking step that assigns filopodia identities across time points. The segmentation pipeline consists of the following seven steps.

\section{Pixel segmentation}

We use the slic segmentation algorithm (Achanta et al., 2012) without enforced connectivity to turn the greyscale frame into a black-and-white segmented frame without blurring. We allow three labels to give the algorithm enough finesse to resolve filopodia and then consider every pixel that the algorithm assigns a label to as foreground.

\section{Cell body identification}

In order to identify cell bodies to mask the cell interior, we employ aggressive gaussian blurring $(\sigma=5)$ together with adaptive thresholding with a large $(999 \times 999$ pixels) gaussian-weighed neighborhood (van der Walt et al., 2014) together with an erosion operation with a 5-pixel disk as stencil. We then use this as a mask to remove parts of the skeleton that lie inside cell bodies.

\section{Vesselness filter}

We employ a modified version of the Frangi Vesselness measure that is popular for tracing blood vessels in MRI data (Frangi et al., 1998). This filter employs the eigenvalues of the Hessian, which describe the local curvature of the image signal. In particular, the smallest of the eigenvalues describes the local 'saddleness'. We therefore calculate the eigenvalues $\lambda_{1}, \lambda_{2}$ and $\lambda_{3}$ of the Hessian for each pixel in the output of the pixel segmentation and order them such that $\left|\lambda_{1}\right|<\left|\lambda_{2}\right|<\left|\lambda_{3}\right|$. We then calculate the derived quantities $r_{a}=\left|\lambda_{2}\right| /\left|\lambda_{3}\right|, r_{b}=\left|\lambda_{1}\right| / \sqrt{\left|\lambda_{2} \lambda_{3}\right|}$ and $S=\sqrt{\lambda_{1}^{2}+\lambda_{2}^{2}+\lambda_{3}^{2}}$. They are then combined into the Vesselness measure

$$
V=\left[1-\exp \left(-4 r_{a}^{2}\right)\right] \exp \left(-4 r_{b}^{2}\right)\left[1-\exp \left(-8 \frac{S^{2}}{\max \{S\}^{2}}\right)\right]
$$

The exponential weights were chosen empirically such that filopodia in test movies were identified correctly.

\section{Vesselness output segmentation}

We again employ the slic segmentation algorithm without enforced connectivity, but a compactness parameter of 10 to identify bright connected lines in the Vesselness filter output and subsequently employ a binary closing step to heal broken lines.

\section{Remove small objects}

In this step, we remove small, connected regions containing less than 200 pixels (note that this step still operates on a $z$-stack of images, hence the rather high pixel number threshold), which stem from noise and not actual filopodia.

\section{Skeletonization}

We use the 3D skeletonization algorithm in Lee et al. (1994) to turn the resulting elongated patches into thin lines. We remove parts of the skeleton that are inside the cell bodies as detected in step 2.

\section{Detection of connected line segments}

We now label and iterate over all the connected regions of the skeleton. Each region has a collection of connected pixel coordinates (forming branched lines). We algorithmically split them into line segments and save them for subsequent manual editing and merging.

Following the automated extraction of filopodial line segments, we manually remove line segments that either were erroneously detected (due to e.g. noise in the image, or from parts of other structures in the field of view) or are part of filopodia stemming from cells other than the LT muscle cells. We also join line segments forming part of the same filopodium.

After the manual editing step, we automatically link and identify filopodia across time points. For each time point, we use the coordinates of all pixels contained in all filopodia over the preceding five time points. For each filopodium in the present time point, we then perform a nextneighbor search to find all previous filopodia that are close (with a cutoff radius of 25 pixels). For each of the candidate matches we calculate the path length difference measure $M_{L}=\left|L_{c}-L_{p}\right| / \max \left\{L_{c}, L_{p}\right\}$ (where $L_{c}$ and $L_{p}$ are the path lengths of the current filopodium and the candidate match filopodium, respectively) and the average direction measure $M_{D}=1-\left|\vec{d}_{c} \cdot \vec{d}_{p} /\left(\left\|\vec{d}_{c}\right\|\left\|\vec{d}_{p}\right\|\right)\right|$ (where $\vec{d}=\sum_{i=2}^{N}\left(\overrightarrow{x_{i}}-\overrightarrow{x_{i}}\right) / N$ is the 
average non-normalized direction vector of a filopodium). We then summarize these measure into a score $S=0.46 M_{L}+0.6 M_{D}$ (where the weights were manually adjusted to yield optimal results) and store the current filopodium and the match candidate together with the score. The pairs are then sorted by their score (lowest to highest) and iteratively linked until no more possible matches can be performed. Any leftover filopodia that were not matched to previously existing filopodia are considered to be newly created. After this step, we save the list of filopodia and their segment coordinates in each frame in which they exist and extract quantities such as the maximum filopodia length (over the lifetime of a filopodium) and the number of filopodia in existence in each movie.

\section{Acknowledgements}

We thank the Gurdon Institute imaging facility for microscopy support, and Jonathan Gadsby and Bishara Marzook for reading the manuscript.

\section{Competing interests}

The authors declare no competing or financial interests.

\section{Author contributions}

Conceptualization: B.R., Y.I., U.D., N.H.B., J.L.G.; Methodology: B.R., Y.I., N.H.B., J.L.G.; Software: U.D.; Validation: B.R., Y.I.; Formal analysis: B.R., U.D.; Investigation: B.R., Y.I., U.D.; Resources: Y.I., J.F., N.H.B.; Data curation: B.R. U.D.; Writing - original draft: Y.I., J.L.G.; Writing - review \& editing: B.R., Y.I., N.H.B. J.L.G.; Visualization: B.R., Y.I.; Supervision: N.H.B., J.L.G.; Project administration: J.L.G.; Funding acquisition: N.H.B., J.L.G.

\section{Funding}

This work was supported by Wellcome Trust grants 069943 and 086451 to N.H.B. European Research Council grant 281971 and Wellcome Trust Research Career Development Fellowship WT095829AIA to J.L.G. We acknowledge the core funding by the Wellcome Trust (092096) and Cancer Research UK (C6946/A14492). U.D. was supported by a Junior Interdisciplinary Fellowship (Wellcome Trust grant No. 105602/Z/14/Z and a Herchel Smith Postdoctoral Fellowship). Deposited in PMC for immediate release.

\section{Supplementary information}

Supplementary information available online at

http://jcs.biologists.org/lookup/doi/10.1242/jcs.217133.supplemental

\section{References}

Achanta, R., Shaji, A., Smith, K., Lucchi, A., Fua, P. and Süsstrunk, S. (2012). SLIC superpixels compared to state-of-the-art superpixel methods. IEEE Trans. Pattern Anal. Mach. Intell. 34, 2274-2282.

Bahri, S. M., Choy, J. M., Manser, E., Lim, L. and Yang, X. (2009). The Drosophila homologue of Arf-GAP GIT1, dGIT, is required for proper muscle morphogenesis and guidance during embryogenesis. Dev. Biol. 325, 15-23.

Bate, M. (1990). The embryonic development of larval muscles in Drosophila. Development 110, 791-804.

Brown, N. H. (1994). Null mutations in the alpha PS2 and beta PS integrin subunit genes have distinct phenotypes. Development 120, 1221-1231.

Bunch, T. A., Salatino, R., Engelsgjerd, M. C., Mukai, L., West, R. F. and Brower, D. L. (1992). Characterization of mutant alleles of myospheroid, the gene encoding the beta subunit of the Drosophila PS integrins. Genetics 132, 519-528.

Callahan, C. A., Bonkovsky, J. L., Scully, A. L. and Thomas, J. B. (1996). derailed is required for muscle attachment site selection in Drosophila. Development 122, 2761-2767.

Chou, T. B. and Perrimon, N. (1996). The autosomal FLP-DFS technique for generating germline mosaics in Drosophila melanogaster. Genetics 144, 1673-1679.

Comer, A. R., Ahern-Djamali, S. M., Juang, J.-L., Jackson, P. D. and Hoffmann, F. M. (1998). Phosphorylation of Enabled by the Drosophila Abelson tyrosine kinase regulates the in vivo function and protein-protein interactions of Enabled. Mol. Cell. Biol. 18, 152-160.

Davenport, R. W., Dou, P., Rehder, V. and Kater, S. B. (1993). A sensory role for neuronal growth cone filopodia. Nature 361, 721-724. de Joussineau, C., Bataille, L., Jagla, T. and Jagla, K. (2012). Diversification of muscle types in Drosophila: upstream and downstream of identity genes. Curr. Top. Dev. Biol. 98, 277-301.

Devenport, D., Bunch, T. A., Bloor, J. W., Brower, D. L. and Brown, N. H. (2007). Mutations in the Drosophila alphaPS2 integrin subunit uncover new features of adhesion site assembly. Dev. Biol. 308, 294-308.

Estrada, B., Gisselbrecht, S. S. and Michelson, A. M. (2007). The transmembrane protein Perdido interacts with Grip and integrins to mediate myotube projection and attachment in the Drosophila embryo. Development 134, 4469-4478.

Frangi, A. F., Niessen, W. J., Vincken, K. L. and Viergever, M. A. (1998) Multiscale vessel enhancement filtering. Lect. Notes Comput. Sci. 1496, 130-137.

Hoefen, R. J. and Berk, B. C. (2006). The multifunctional GIT family of proteins. J. Cell Sci. 119, 1469-1475.

Jacquemet, G., Hamidi, H. and Ivaska, J. (2015). Filopodia in cell adhesion, 3D migration and cancer cell invasion. Curr. Opin. Cell Biol. 36, 23-31.

Klapholz, B., Herbert, S. L., Wellmann, J., Johnson, R., Parsons, M. and Brown, N. H. (2015). Alternative mechanisms for talin to mediate integrin function. Curr. Biol. 25, 847-857.

Kramer, S. G., Kidd, T., Simpson, J. H. and Goodman, C. S. (2001). Switching repulsion to attraction: changing responses to slit during transition in mesoderm migration. Science 292, 737-740.

Lee, T. C., Kashyap, R. L. and Chu, C. N. (1994). Building skeleton models via 3-D medial Surface axis thinning algorithms. CVGIP, Graph. Models Image Process. 56, $462-478$.

Loureiro, J. J., Rubinson, D. A., Bear, J. E., Baltus, G. A., Kwiatkowski, A. V. and Gertler, F. B. (2002). Critical roles of phosphorylation and actin binding motifs, but not the central proline-rich region, for Ena/vasodilator-stimulated phosphoprotein (VASP) function during cell migration. Mol. Biol. Cell 13, 2533-2546.

Maartens, A. P. and Brown, N. H. (2015). The many faces of cell adhesion during Drosophila muscle development. Dev. Biol. 401, 62-74.

Martin-Bermudo, M. D. and Brown, N. H. (1999). Uncoupling integrin adhesion and signaling: the $\beta P S$ cytoplasmic domain is sufficient to regulate gene expression in the Drosophila embryo. Genes Dev. 13, 729-739.

Mendoza, M. C. (2013). Phosphoregulation of the WAVE regulatory complex and signal integration. Semin. Cell Dev. Biol. 24, 272-279.

Newsome, T. P., Schmidt, S., Dietzl, G., Keleman, K., Åsling, B., Debant, A. and Dickson, B. J. (2000). Trio combines with dock to regulate Pak activity during photoreceptor axon pathfinding in Drosophila. Cell 101, 283-294.

Robertson, H. M., Preston, C. R., Phillis, R. W., Johnson-Schlitz, D. M., Benz, W. K. and Engels, W. R. (1988). A stable genomic source of $P$ element transposase in Drosophila melanogaster. Genetics 118, 461-470.

Rochlin, K., Yu, S., Roy, S. and Baylies, M. K. (2010). Myoblast fusion: when it takes more to make one. Dev. Biol. 341, 66-83.

Ruiz-Gómez, M., Romani, S., Hartmann, C., Jackle, H. and Bate, M. (1997). Specific muscle identities are regulated by Kruppel during Drosophila embryogenesis. Development 124, 3407-3414.

Schnorrer, F. and Dickson, B. J. (2004). Muscle building; mechanisms of myotube guidance and attachment site selection. Dev. Cell 7, 9-20.

Schnorrer, F., Kalchhauser, I. and Dickson, B. J. (2007). The transmembrane protein Kon-tiki couples to Dgrip to mediate myotube targeting in Drosophila Dev. Cell 12, 751-766.

Schweitzer, R., Zelzer, E. and Volk, T. (2010). Connecting muscles to tendons: tendons and musculoskeletal development in flies and vertebrates. Development 137, 2807-2817.

Tanentzapf, G., Martin-Bermudo, M. D., Hicks, M. S. and Brown, N. H. (2006). Multiple factors contribute to integrin-talin interactions in vivo. J. Cell Sci. $119,1632-1644$

van der Walt, S., Schönberger, J. L., Nunez-Iglesias, J., Boulogne, F., Warner, J. D., Yager, N., Gouillart, E., Yu, T. and The scikit-image contributors (2014). scikit-image: image processing in Python. PeerJ 2, e453.

Weitkunat, M., Kaya-Çopur, A., Grill, S. W. and Schnorrer, F. (2014). Tension and force-resistant attachment are essential for myofibrillogenesis in Drosophila flight muscle. Curr. Biol. 24, 705-716.

Weitkunat, M., Brasse, M., Bausch, A. R. and Schnorrer, F. (2017). Mechanical tension and spontaneous muscle twitching precede the formation of cross-striated muscle in vivo. Development 144, 1261-1272.

Zanet, J., Jayo, A., Plaza, S., Millard, T., Parsons, M. and Stramer, B. (2012). Fascin promotes filopodia formation independent of its role in actin bundling J. Cell Biol. 197, 477-486.

Zeng, F.-M., Wang, X.-N., Shi, H.-S., Xie, J.-J., Du, Z.-P., Liao, L.-D., Nie, P.-J. Xu, L.-Y. and Li, E.-M. (2017). Fascin phosphorylation sites combine to regulate esophageal squamous cancer cell behavior. Amino Acids 49, 943-955. 